\title{
SARS-CoV-2-Induced ARDS Associates with MDSC Expansion, Lymphocyte Dysfunction, and Arginine Shortage
}

\author{
Florian Reizine $^{1,2,3}$. Mathieu Lesouhaitier ${ }^{1,2,3}$ - Murielle Gregoire ${ }^{2,3} \cdot$ Kieran Pinceaux $^{1} \cdot$ Arnaud Gacouin $^{1}$. \\ Adel Maamar ${ }^{1}$ • Benoit Painvin ${ }^{1}$. Christophe Camus ${ }^{1} \cdot$ Yves Le Tulzo $^{1}$ • Pierre Tattevin ${ }^{1}$ - Matthieu Revest ${ }^{1}$. \\ Audrey Le Bot ${ }^{1}$. Alice Ballerie ${ }^{4}$. Berengère Cador-Rousseau ${ }^{4}$ - Mathieu Lederlin ${ }^{5} \cdot$ Thomas Lebouvier $^{6}$. \\ Yoann Launey ${ }^{6}$ - Maelle Latour ${ }^{2,3}$. Clotilde Verdy ${ }^{2,3}$. Delphine Rossille ${ }^{2,3}$. Simon Le Gallou ${ }^{2,3}$. Joelle Dulong ${ }^{2,3}$. \\ Caroline Moreau $^{7} \cdot$ Claude Bendavid $^{7} \cdot$ Mikael Roussel $^{2,3} \cdot$ Michel Cogne $^{2,3} \cdot$ Karin Tarte $^{2,3} \cdot$ Jean-Marc Tadié $^{1,2,3,8}$ (D)
}

Received: 1 August 2020 / Accepted: 12 November 2020 / Published online: 2 January 2021

(C) Springer Science+Business Media, LLC, part of Springer Nature 2021

\begin{abstract}
Purpose The SARS-CoV-2 infection can lead to a severe acute respiratory distress syndrome (ARDS) with prolonged mechanical ventilation and high mortality rate. Interestingly, COVID-19-associated ARDS share biological and clinical features with sepsis-associated immunosuppression since lymphopenia and acquired infections associated with late mortality are frequently encountered. Mechanisms responsible for COVID-19-associated lymphopenia need to be explored since they could be responsible for delayed virus clearance and increased mortality rate among intensive care unit (ICU) patients.

Methods A series of 26 clinically annotated COVID-19 patients were analyzed by thorough phenotypic and functional investigations at days 0,4 , and 7 after ICU admission.

Results We revealed that, in the absence of any difference in demographic parameters nor medical history between the two groups, ARDS patients presented with an increased number of myeloid-derived suppressor cells (MDSC) and a decreased number of $\mathrm{CD}^{\text {pos }}$ effector memory cell compared to patients hospitalized for COVID-19 moderate pneumonia. Interestingly, COVID-19-related MDSC expansion was directly correlated to lymphopenia and enhanced arginase activity. Lastly, T cell proliferative capacity in vitro was significantly reduced among COVID-19 patients and could be restored through arginine supplementation.

Conclusions The present study reports a critical role for MDSC in COVID-19-associated ARDS. Our findings open the possibility of arginine supplementation as an adjuvant therapy for these ICU patients, aiming to reduce immunosuppression and help virus clearance, thereby decreasing the duration of mechanical ventilation, nosocomial infection acquisition, and mortality.
\end{abstract}

Keywords Covid-19 · ARDS · MDSC $\cdot$ Lymphocytes · Arginine $\cdot$ Cross infection · Immunosuppression

Florian Reizine and Mathieu Lesouhaitier contributed equally to this work.

Jean-Marc Tadié

jeanmarc.tadie@chu-rennes.fr

1 Infectious Diseases and Intensive Care Unit, Pontchaillou University Hospital, 2 rue Henri Le Guilloux, 35033 Rennes, France

2 SITI, Pole de Biologie, Pontchaillou University Hospital, 2 rue Henri Le Guilloux, 35033 Rennes, France

3 UMR 1236, University of Rennes, INSERM, Etablissement Français du Sang Bretagne, Rennes, France

4 Department of Internal Medicine and Clinical Immunology, Pontchaillou University Hospital, 2 rue Henri Le Guilloux, 35033 Rennes, France

5 Department of Radiology, Pontchaillou University Hospital, 2 rue Henri Le Guilloux, 35033 Rennes, France Hospital, 2 rue Henri Le Guilloux, 35033 Rennes, France

7 Department of Biochemistry, Pontchaillou University Hospital, 2 rue Henri Le Guilloux, 35033 Rennes, France

8 Service des Maladies Infectieuses et Réanimation Médicale, Hôpital Pontchaillou, 2 rue Henri Le Guilloux, 35033 Rennes Cedex 9, France 


\section{Introduction}

Coronavirus disease 2019 (COVID-19) is an infectious disease caused by severe acute respiratory syndrome coronavirus 2 (SARS-CoV-2) which can lead to severe pneumonia with acute respiratory distress syndrome (ARDS), responsible for hospitalizations in intensive care unit (ICU) with an ICU mortality rate reaching 30 to $60 \%$ [1, 2]. Among the factors associated with this severe outcome, older age, comorbid conditions, and an elevated body mass index have been constantly reported [1-4]. Furthermore, lymphopenia and diminished viral clearance illustrated by persistent SARS-CoV-2 in the lung have been associated with COVID-19 severity and poor prognosis [5-7]. Viral clearance and the resolution of infection involve a complex immune response initiated by resident cells of the respiratory tract along with innate immune cells and is ultimately resolved by adaptive immune cells. Like SARSCoV-1, SARS-CoV-2 uses ACE2 as a receptor for bronchial epithelium cellular entry and activates inflammation through multiple pathways, directly related to cytotoxicity and dependent on innate immune cells $[8,9]$. Although local immune responses have not yet been explored in COVID-19, it is known in severe respiratory viral infections that $\mathrm{T}$ cells crucially contribute to virus clearance from infected lungs and thus to a favorable outcome $[5,10]$. In addition to delaying virus clearance, lymphopenia and/or functional $\mathrm{T}$ cell defects also favor secondary infections by opportunistic pathogens inducing prolonged mechanical ventilation responsible for late mortality $[1,11]$. While sepsis is more often associated with bacterial or fungal infections [12], COVID-19 can thus feature "viral sepsis" with a pathologic host response characterized by an important cytokine release, and critically ill associated immunosuppression rather than "typical" ARDS [13, 14]. Lymphopenia, a crucial component of severe COVID-19, has in fact been noted in other severe respiratory viral infections, notably H1N1 influenza [15]. More broadly, immunosuppression in critically ill patients has been shown to be associated with increased lymphocyte apoptosis, decreased lymphocyte proliferation, and the striking emergence of myeloid-derived suppressor cells (MDSC), all associated with poorer outcome and nosocomial infections in ICU [16-18]. The underlying mechanisms of this MDSC-associated immunosuppression in infectious diseases are still not fully understood. However, various aspects of MDSC-mediated T cell immunosuppression have been reported in other conditions where MDSC are amplified, such as cancers and obesity [19]. Moreover, during acute viral illness, MDSC have been associated with chronicity in viral infections and poor clinical outcome [20]. Whether and how MDSC, including monocytic (M-MDSC) and granulocytic (G-MDSC) subsets, might be involved in the pathogenesis of SARS-Cov-2 has not yet been fully explored although emerging data reported that MDSC might influence disease severity $[21,22]$.
The present study explores the potential relationship between MDSC expansion, lymphopenia, and lymphocyte dysfunction in the first week following admission to ICU among COVID-19-associated ARDS patients.

\section{Materials and Methods}

\section{Study Design, Patients, and Samples}

This study was performed in the department of infectious diseases and the intensive care unit (ICU) at Rennes University Hospital. The study design was approved by our ethic committee (CHU Rennes, $\left.n^{\circ} 18.101-2\right)$, and informed consent was obtained from the patients. From late March 2020 to May 2020, 26 consecutive patients with moderate and severe acute respiratory distress syndrome (ARDS) or moderate pneumonia related to SARS-CoV2 infection were prospectively enrolled and compared with 13 healthy donors. Pregnant women, patients younger than 18 years old, patients with malignancy, HIV-infected patients, and patients with preexisting immune disorders or receiving immunosuppressive agents were excluded. The presence of SARS-CoV-2 in respiratory specimens (nasal and pharyngeal swabs or sputum) was detected by real-time reverse transcriptionpolymerase chain reaction (RT-PCR) methods. COVID-19 moderate pneumonia was defined as cases exhibiting fever and respiratory symptoms with radiological findings of pneumonia. Severe and moderate ARDS were defined in accordance with international guidelines [23]. The following data were recorded: age, reason for admission, patient's preexisting condition (current smoking, diabetes, hypertension, cardiovascular disease, pulmonary disease, liver disease), obesity (defined as a body mass index $>30 \mathrm{~kg} / \mathrm{m}^{2}$ ), ICU and hospital length of stay, mortality, the need for invasive mechanical ventilation, the need for prone position ventilation, the need for renal replacement therapy, and the need for vasoconstrictor agent during ICU stay. The occurrence of nosocomial infections was also recorded $[17,24]$. Initial clinical laboratory measurement included a complete blood count and a serum biochemical test. Lymphopenia was defined as an absolute lymphocyte count under 1.5 cells $/ \mu \mathrm{L} \times 10^{3}$. The Simplified Acute Physiology Score (SAPS II) and the Sequential Organ Failure Assessment (SOFA) score at admission to ICU were used to assess severity $[25,26]$. A senior radiologist who was blinded to the other clinical information reviewed the CT chest scans and classified lung injury as absent or minimal $(<10 \%)$, moderate $(10-15 \%)$, extensive $(25-50 \%)$, or severe $(50-75 \%)$. Blood samples were collected within the first $24 \mathrm{~h}$ following admission, 4 days (day 4 ) after admission and 7 days (day 7) after admission when patients were still hospitalized. The time lapse between sampling and initiation of laboratory procedures was less than $1 \mathrm{~h}$. Peripheral blood mononuclear 
cells (PBMC) were isolated by Ficoll density gradient, and plasma samples were stored at $-80{ }^{\circ} \mathrm{C}$ until use.

\section{Flow Cytometry}

Quantification of monocytic populations was performed on fresh whole blood. After staining, erythrocytes were lysed twice with EasyLyse (Dako, Glostrup, Denmark) before washing in PBS. Monocyte populations have been determined based on CD14 and $\mathrm{CD} 16$ expression. M-MDSC were defined as $\mathrm{CD} 14^{\text {pos }}$ HLA-DR ${ }^{\text {low }}$ cells. Expression of PD-L1 has been determined on three monocyte populations $\left(\mathrm{CD} 14^{\text {pos }} \mathrm{CD} 16^{\text {neg }}, \mathrm{CD} 14^{\text {pos }}\right.$ $\mathrm{CD} 16^{\mathrm{pos}}$, and $\left.\mathrm{CD} 14^{\mathrm{low}} \mathrm{CD} 16^{\mathrm{pos}}\right)$. G-MDSC and early-MDSC (e-MDSC) were defined on fresh PBMC isolated after Ficoll density gradient and were defined as $\mathrm{CD} 45^{\text {pos }} \mathrm{Lin}^{\text {neg }} \mathrm{HLA}$ $\mathrm{DR}^{1 \mathrm{low}} \mathrm{CD} 15^{\text {pos }} \mathrm{CD} 11 \mathrm{~b}^{\text {pos }}$ for G-MDSCs and as $\mathrm{CD} 45^{\text {pos }} \mathrm{CD} 3{ }^{\text {neg }} \mathrm{Lin}^{\text {neg }} \mathrm{HLA}-\mathrm{DR}{ }^{\text {low }} \mathrm{CD} 15^{\text {pos }} \mathrm{CD} 33^{\text {pos }}$ for eMDSC. Gating schemes for the monocyte populations and representative staining panels of the MDSC populations are represented in the supplementary Figs. 1 and 2. Precision Count Beads (Biolegend, San Diego, CA) were added after staining to calculate the absolute number of cell subpopulations. B cells, NK cells, and $\mathrm{T}$ cells were analyzed on whole blood by using CD19, CD3, CD56, and CD16. Frequencies of cells were defined by flow cytometry, and absolute counts were calculated relative to the lymphocyte count defined on a cell counter before processing. Naïve $(\mathrm{N})$, central memory $(\mathrm{CM})$, effector memory (EM), and effector memory expressing CD45RA (EMRA) were defined based on CCR7 and CD45RA expression, on CD4 and CD8 cells. On each cell types, the expression of HLA-DR and PD-1 was analyzed. All antibodies and fluorochromes are summarized in supplementary Table 1. A Fortessa X20 flow cytometer (Becton Dickinson, San Jose, CA, USA) was used to run all samples. Data were analyzed using Kaluza 2.0 software (Beckman Coulter, Brea, CA, USA).

\section{Apoptotic Analysis}

Whole blood was incubated with CD3 BV421 (Becton Dickinson). Erythrocytes were then lysed twice using Easylyse (Dako, Glostrup, Denmark). To evaluate the proportion of apoptotic T cells, PE-conjugated active caspase-3 apoptosis kit (Becton Dickinson) has been used. Samples were run in a Fortessa X20 flow cytometer (Becton Dickinson) and data were analyzed using Kaluza 2.0 software (Beckman Coulter).

\section{T Cell Proliferation}

PBMC isolated after Ficoll density gradient were labeled with carboxyfluorescein succinimidyl ester (CFSE, $200 \mathrm{nM}$; interchim, Montluçon, France). The quantification of T cells was obtained by staining PBMC with an anti-CD3 APC antibody (Becton Dickinson). PBMC were seeded in 96well round-bottom plates at a concentration of $1 \times 10^{5} \mathrm{~T}$ cells per well. Cells were cultured in RPMI 1640 supplemented with $10 \%$ human $\mathrm{AB}$ serum (Biowest, Nuaillé, France) and anti-CD3 and anti-CD28 monoclonal antibodies $(0.6 \mu \mathrm{g} / \mathrm{mL}$, Sanquin, Amsterdam, Netherlands). When indicated, cells were cultured in presence of the following chemical inhibitors or their controls: (i) L-arginine (1 mM, Sigma-Aldrich, St Louis, MO, USA) or with control D-arginine; (ii) coptisine (an IDO inhibitor) (50 nM, Sigma-Aldrich, St Louis, MO, USA) or vehicle; and (iii) PD-L1 blocking antibodies (10 $\mu \mathrm{M}$, ebioscience, San Diego, CA, USA) or IgG. After 4 days of culture, cells were harvested and labeled with FVS 780 (Becton Dickinson) for cell viability, CD2 PC7 and CD8 APC (Beckman Coulter), CD4 BUV496 and CD14 BV605 (Becton Dickinson). CFSE dilution was assessed on FVS ${ }^{\text {neg }}$ viable T cells by flow cytometry on Fortessa X20 (Becton Dickinson) and results were analyzed with ModFit LT software (Verity Software, Topsham, ME).

\section{Cytokine and Amino Acid Quantifications}

Plasma interleukin (IL)-6, IL-10, chemokine (C-C motif) ligand 2 (CCL2), chemokine (C-X-C motif) ligand 9 (CXCL9), CXCL10, and granulocyte-colony stimulating factor (G-CSF) were measured using a Milliplex map magnetic bead kit (EMD Millipore). Indoleamine 2,3-dioxygenase (IDO) activity was evaluated by measuring kynurenine and tryptophan levels and arginase activities by measuring ornithine and arginine concentrations by liquid chromatography coupled with tandem mass spectrometry (LC MS-MS).

\section{Statistical Analysis}

Continuous variables were expressed as median (interquartile range, IQR) and compared using the nonparametric MannWhitney $U$ test or the Kruskal-Wallis test or the Wilcoxon matched-pairs for matched samples as appropriate. Dunn's correction tests were used if multiple comparisons were requested. Qualitative data were compared using chi-square test or Fisher exact test when required. Correlations between two continuous variables were investigated using the Pearson's rank test. Tests were two-sided with significance set at $\alpha$ less than 0.05. All data were analyzed with GraphPad Prism 8.4 (GraphPad Software, La Jolla, CA).

\section{Results}

\section{Study Population}

Twenty-six COVID-19 patients were included in this prospective study (13 with ARDS and 13 with moderate pneumonia). 
Representative CT scans are shown in Fig. 1 and their demographic and baseline characteristics in Table 1. Importantly, there was no difference in demographic characteristics, including comorbidities classically associated with COVID-19 severity, nor was there any difference in duration of symptoms before inclusion between the two groups of patients, thus making it possible to focus our analysis on the biological parameters related to COVID severity. Complete clinical characteristics at day 0 , day 4 and day 7 are summarized in the supplementary Table 2 .

Of note, patients admitted in ICU received selected digestive decontamination as already described [27]. In addition, the patients received 3 rd-generation cephalosporin for 1 week. None received corticosteroids or experimental treatments.

\section{Lymphopenia, Accumulation of Circulating MDSC, and Susceptibility to Nosocomial Infections}

To determine the systemic impact of SARS-COV-2 on immune cells, a complete blood count was performed first. An increase in leukocyte counts at day 4 and day 7 along with an increased number of circulating neutrophils was noted among COVID-19 ARDS patients compared to patients with moderate pneumonia (Fig. 2A). By contrast, lymphopenia was observed at admission for both groups of COVID-19 patients and persisted until day 7 only for patients with ARDS (Fig. 2A). This persistent lymphopenia was specifically related to a $\mathrm{T}$ cell count decrease whereas NK and B cell counts were not significantly affected (Fig. 2A). Conversely, circulating monocytes were early and transiently reduced specifically among ARDS patients. Platelets counts were not affected by clinical status. By flow cytometry, we defined CD4 and CD8 effector memory $T$ cells to be decreased at day 4 and/or day 7 while other CD4 and CD8 subsets were unaffected (Fig. 2B and Supplementary Fig. 3). As in previous reports [6], all of our patients exhibited lymphopenia at the time of admission, predominantly due to a CD8 effector memory $\mathrm{T}$ cell defect (CD8 EM). This anomaly persisted during the first week after admission among ARDS patients.

In parallel, M-MDSC and G-MDSC were both enriched among moderate and severe COVID patients at day 0 and remained high until day 7 only in ARDS patients thus

Table 1 Demographic and baseline characteristics

\begin{tabular}{|c|c|c|c|c|}
\hline & All patients $(n=26)$ & $\operatorname{ARDS} \operatorname{COVID}(n=13)$ & Moderate COVID $(n=13)$ & $P$ value \\
\hline \multicolumn{5}{|l|}{ Demographic characteristics } \\
\hline Age, years & $55(47-67)$ & $58(53-67)$ & $54(45-67)$ & 0.19 \\
\hline $\begin{array}{l}\text { Sex } \\
\text { Men }\end{array}$ & $\begin{array}{l}18(69) \\
8(31)\end{array}$ & $\begin{array}{l}10(77) \\
3(23)\end{array}$ & $\begin{array}{l}8(61) \\
5(39)\end{array}$ & 0.67 \\
\hline \multicolumn{5}{|l|}{ Women } \\
\hline Current smoking & 0 & 0 & 0 & $>0.99$ \\
\hline \multicolumn{5}{|l|}{ Coexisting conditions } \\
\hline Any & $15(58)$ & $7(54)$ & $8(61)$ & $>0.99$ \\
\hline Diabetes & $4(15)$ & $3(23)$ & $1(8)$ & 0.59 \\
\hline Hypertension & $9(35)$ & $5(38)$ & $4(31)$ & 0.22 \\
\hline Cardiovascular disease & $3(11)$ & $3(23)$ & 0 & $>0.99$ \\
\hline Obesity (BMI > 30) & $9(35)$ & $4(31)$ & $5(38)$ & $>0.99$ \\
\hline \multicolumn{5}{|c|}{ Clinical and biological baseline characteristics } \\
\hline Days from illness onset to dyspnea & $10(8-12)$ & $10(7-13)$ & $10(8-11)$ & 0.989 \\
\hline Ratio of $\mathrm{PaO}_{2}$ to $\mathrm{F}_{\mathrm{i}} \mathrm{O}_{2}$ & $214(139-362)$ & $140(110-202)$ & $357(250-444)$ & $<0.0001$ \\
\hline SAPS II score on day 1 & $26(17-35)$ & $33(19-39)$ & $22(13-28)$ & 0.11 \\
\hline SOFA score on day 1 & $2(1-9.5)$ & $9(2-10)$ & $1(0-1)$ & $<0.0001$ \\
\hline \multicolumn{5}{|l|}{ Outcomes } \\
\hline Secondary infections & $7(27)$ & $7(54)$ & 0 & 0.007 \\
\hline Vasoconstrictive agents & $7(27)$ & $7(54)$ & 0 & 0.007 \\
\hline \multicolumn{5}{|l|}{$\mathrm{CT}$ analysis scoring } \\
\hline Moderate $(10-15 \%)$ & $4(15)$ & 0 & $4(31)$ & 0.003 \\
\hline Extent $(25-50 \%)$ & $15(58)$ & $6(46)$ & $9(69)$ & \\
\hline Severe $(50-75 \%)$ & $7(27)$ & $7(54)$ & 0 & \\
\hline
\end{tabular}

Data are presented as median (IQR), $n(\%), P$ values comparing ARDS and moderate pneumonia cases are tested by Mann-Whitney $U$ test (continuous variables) or chi-square test (categorical variables). COVID-19 coronavirus disease; $B M I$ body mass index; IQR interquartile range; $P a O 2$ arterial oxygen tension; SAPS II Simplified Acute Physiology Score II; SOFA Sequential Organ Failure Assessment; CT computed tomography 
Fig. $1 \mathrm{CT}$ scan from a patient hospitalized for SARS-CoV-2 induced ARDS at admission (A) and 7 days after $(\mathbf{C})$. CT scan from a patient hospitalized for SARS-CoV-2-induced moderate pneumonia at admission (B) and 9 days after (D)
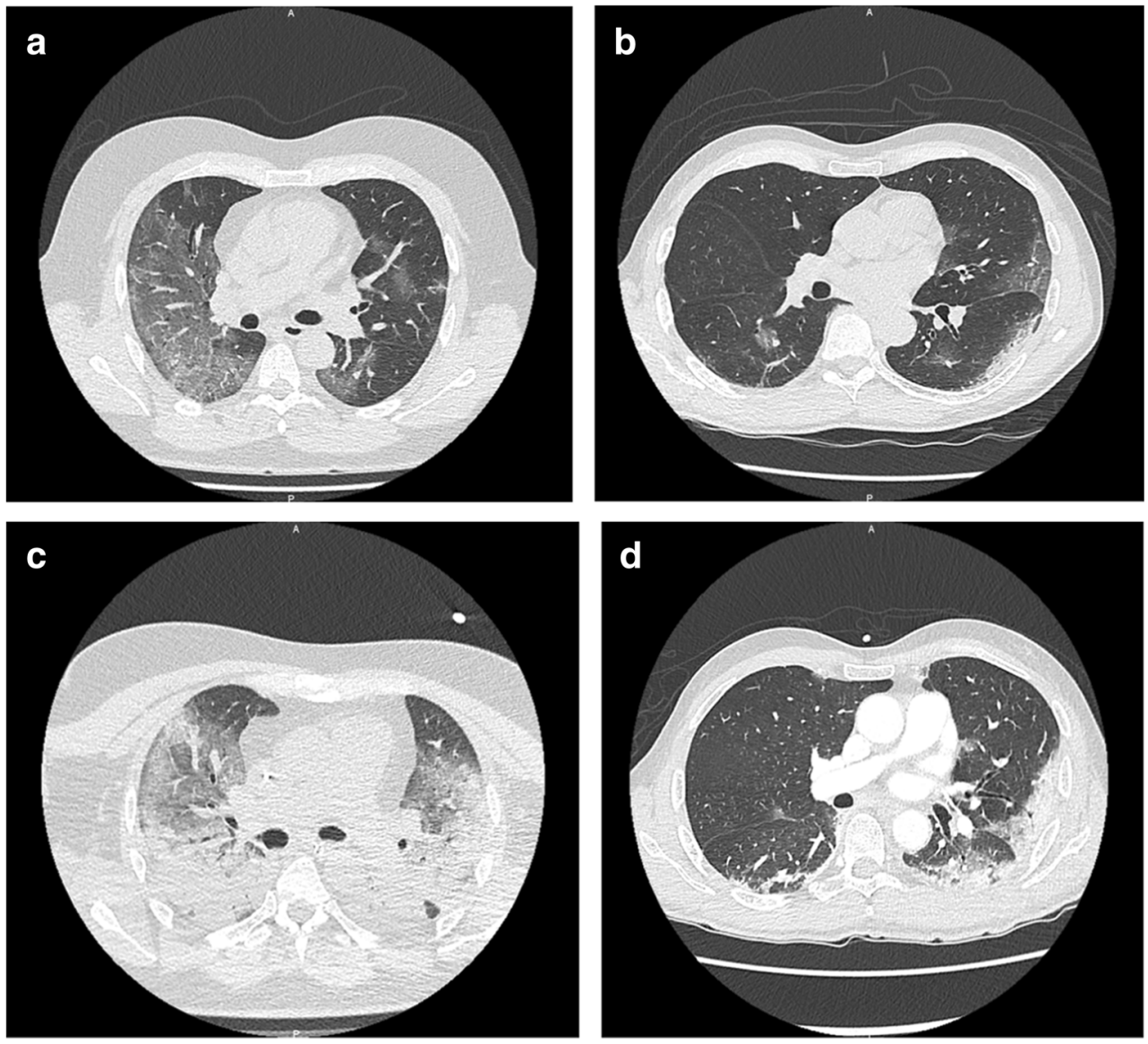

mirroring $\mathrm{T}$ cell lymphopenia (Fig. 2C). Interestingly, MMDSC and G-MDSC were inversely correlated with $\mathrm{T}$ cell count suggesting that they could contribute to $\mathrm{T}$ cell dysfunction. Of note, circulating early-stage MDSC (e-MDSC) were not affected by the ARDS status (data not shown). Among the ARDS patients, seven were diagnosed with ventilatorassociated pneumonia (VAP) related to infection with Aspergillus fumigatus $(n=5)$, herpes simplex virus $(n=4)$, and Enterococcus faecalis $(n=1)$. Noteworthy, VAP occurred after several days in ICU since median duration of mechanical ventilation at infection was 6 days (2-10). This subgroup of ARDS patients with secondary infections had a more marked lymphopenia already detectable on the day of admission and a higher M-MDSC count at day 7 (Fig. 2D). Conversely, GMDSC were not associated with secondary infection. Altogether, these data indicate that COVID-19 was significantly associated with lymphopenia at admission, mostly affecting CD8 EM cells and to a lesser extent CD4 EM cells and that $\mathrm{T}$ cell defect was correlated with M-MDSC expansion and persists in patients with ARDS until day 7.

\section{Cytokines Analysis}

Since cytokine release syndrome (CRS) is a major challenge in COVID-19 [5], various plasma cytokine levels were evaluated in all our COVID-19 patients compared to Healthy Donors (HD) (Fig. 3). Several factors were increased in all COVID-19 patients, but to significantly higher levels in the ARDS group: this included proinflammatory IL-6 and anti-inflammatory IL-10, together with chemokines CCL2, CXCL10, and CXCL9. Finally, only ARDS patients showed increased G-CSF.

\section{COVID-19 Is Associated with a Significant Decrease of Arginine Concentration; Its Supplementation Restores In Vitro T Cells' Ability to Proliferate}

The MDSC increase prompted us to evaluate the activity of enzymes known to support their immunosuppressive activity. Indoleamine 2,3-dioxygenase (IDO) is known to be expressed in M-MDSC [19]. Accordingly, enhanced IDO activity was found among COVID-19 patients, and it was predominant among patients with ARDS and correlated with lymphopenia (Fig. 4A). Additionally, we measured arginase activity, which is expressed by e-MDSC, G-MDSC, and M-MDSC [28]. Our results showed a prolonged decrease in plasma arginine levels along with enhanced arginase activity among COVID patients, particularly in the most severe forms. As for IDO, a correlation between arginase activity and lymphopenia was observed (Fig. 4A). Both IDO and arginase activity could negatively have 


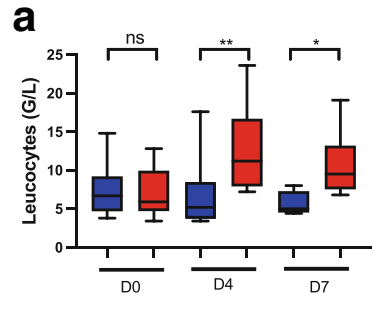

๑ Moderate Pneumonia $\square$ ARDS

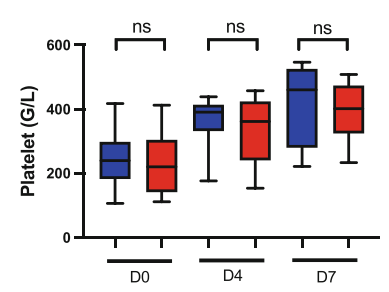

घoderate Pneumonia $\square$ ARDS

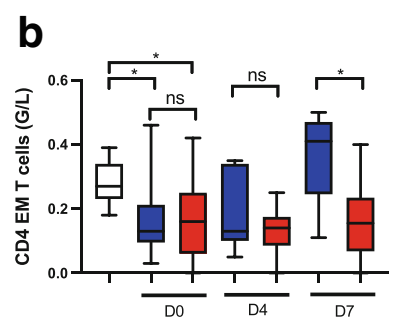

$\square$ HD $\square$ Moderate Pneumonia $\quad$ ARDS

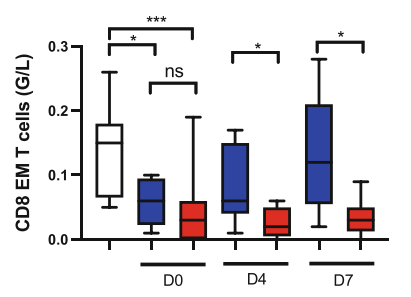

$\square$ HD $\quad$ Moderate Pneumonia $\square$ ARDS

d

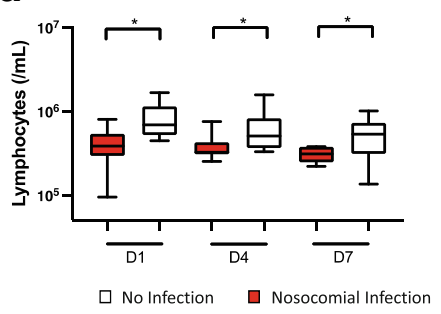

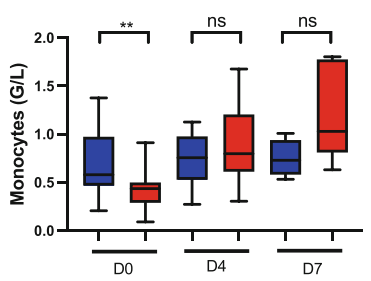

घ Moderate Pneumonia $\square$ ARDS

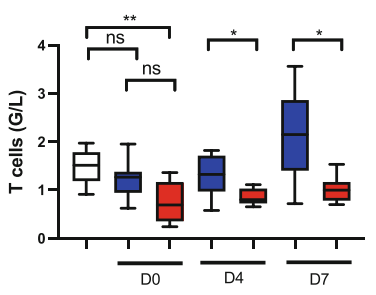

$\square$ HD Moderate Pneumonia $\square$ ARDS

$\square$ HD $\square$ Moderate Pneumonia $\square$ ARDS

C

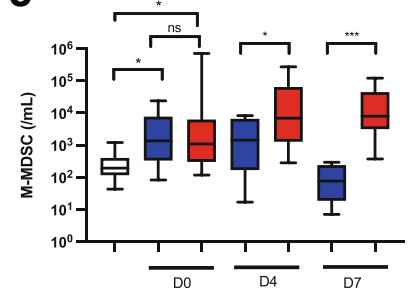

$\square$ HD $\square$ Moderate Pneumonia $\square$ ARDS
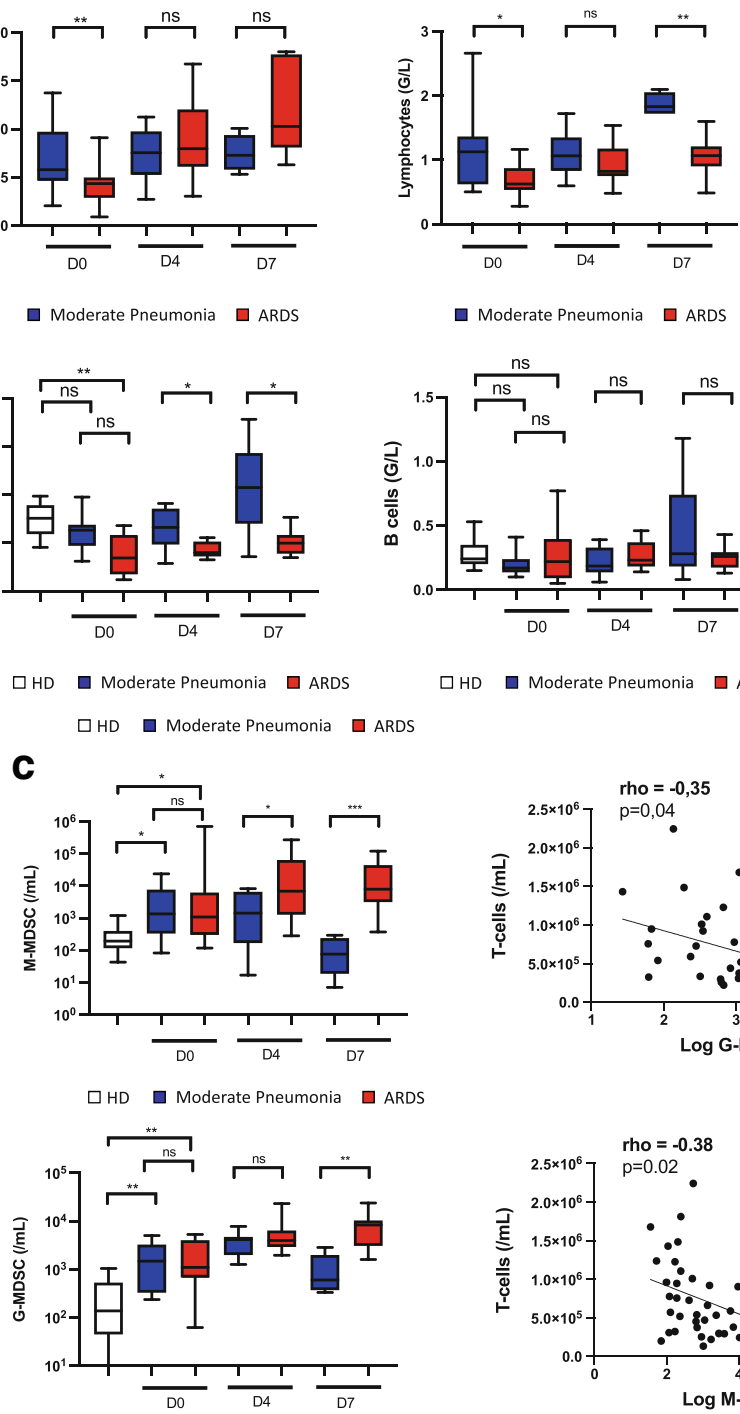

— Moderate Pneumonia $\square$ ARDS

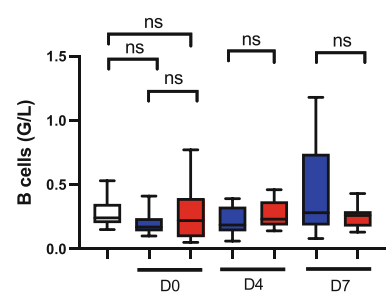

口HD $\quad$ Moderate Pneumonia $\square$ ARDS
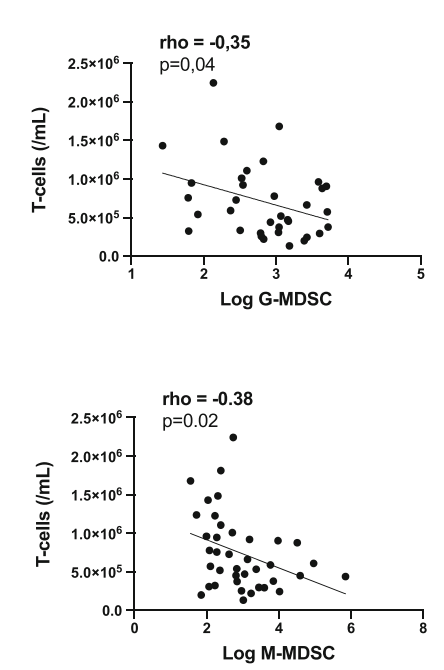

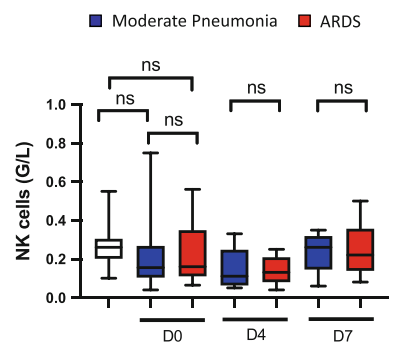

口HD $\quad$ Moderate Pneumonia $\quad$ ARDS
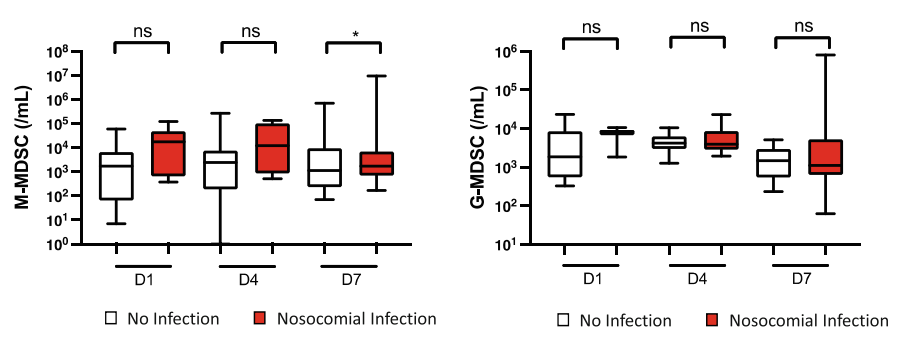

Fig. 2 SARS-CoV-2-induced acute respiratory distress syndrome (ARDS) is associated with lymphopenia and an accumulation of circulating myeloid-derived suppressor cells (MDSC) leading to a higher susceptibility to nosocomial infections. A Blood count from 13 patients hospitalized for SARS-CoV-2 moderate pneumoniae (MP) and 13 patients hospitalized for SARS-CoV-2 ARDS (ARDS) $24 \mathrm{~h}$ after their admission (D0), 4 days after (D4), and 7 days after (D7) and lymphocytes subsets defined by flow cytometry from 13 healthy donors (HD), 13 patients hospitalized for SARS-CoV-2 MP, and 13 patients hospitalized for SARS-CoV-2 ARDS. B CD4 and CD8 effector memory (EM) T cell numeration by flow cytometry. C Peripheral monocytic-MDSC (MMDSC) and granulocytic-MDSC (G-MDSC) recruitment among ARDS patients, moderate COVID cases, and HD. D Two groups were defined

according to the presence or absence of a nosocomial infection. Lymphocyte count, M-MDSC, and G-MDSC recruitment according to the acquisition of nosocomial infection. Nosocomial infections as defined by the Centers for Disease Control and Prevention were screened among patients hospitalized for a SARS-CoV-2 infection over 28 days after their admission. Box and whiskers plot features are as follows: central line in the box is the median, bottom line of the box is first quartile (25\%), and top line of box is third quartile (75\%). Bottom of whiskers is minimum value; top of whiskers is maximum value. Groups were compared using Kruskal Wallis test with Dunn's multiple comparison test (A, B, and C) or Mann-Whitney $U$ test (A and $\mathbf{D}$ ) as appropriate. Pearson correlation coefficients (rho) and $P$ values are indicated for each correlation $(\mathbf{C})$. $* P<0.05 ; * * P<0.01 ; * * * P<0.001$ 


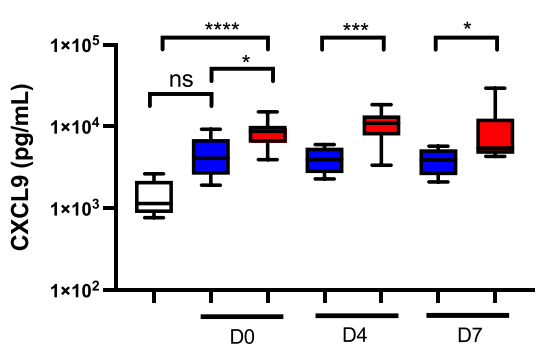

$\square \mathrm{HD}$

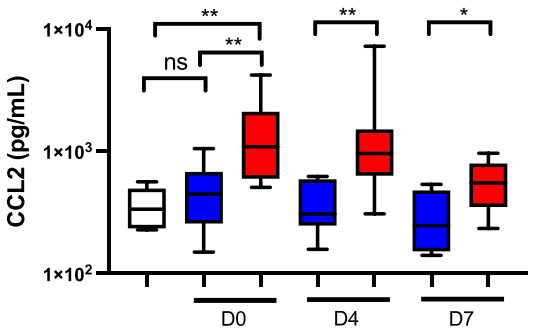

$\square$ HD $\square$ Moderate Pneumonia

$\square$ ARDS

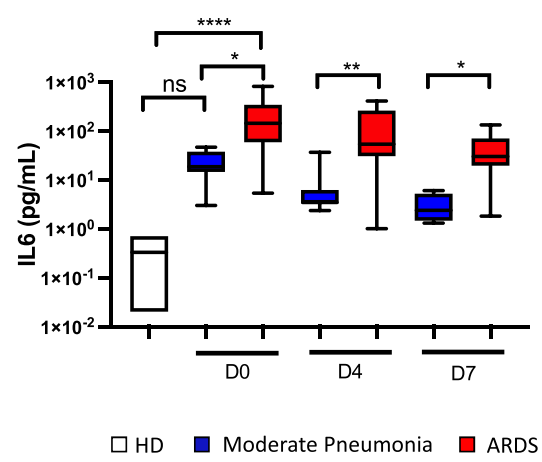

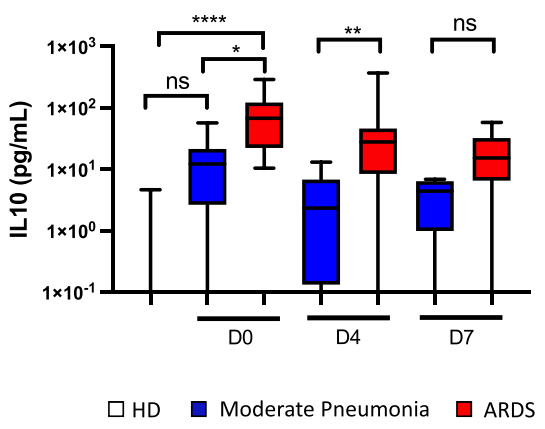

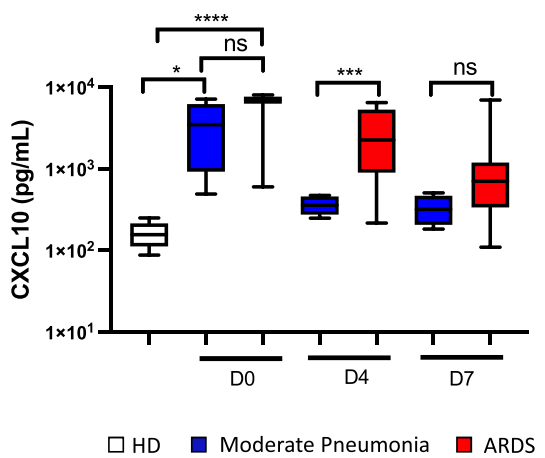

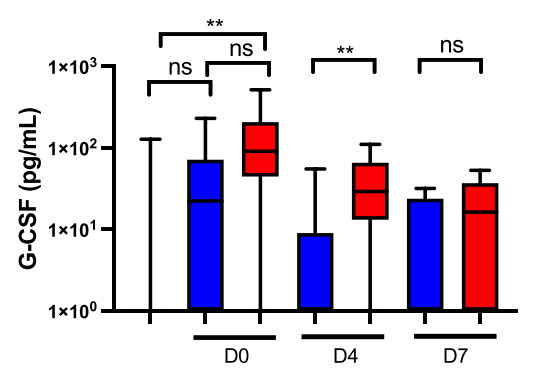

$\square \mathrm{HD}$

Fig. 3 SARS-CoV2 ARDS induces a significant increase among inflammatory, chemoattractant, and immunosuppressive cytokines. MDSC recruiting (IL-6, CCL2, G-CSF), chemoattractant (CXCL9, CXCL10, CCL2), inflammatory (IL-6), and immunosuppressive (IL10) cytokine assessments were performed among the ARDS patients (ARDS), the moderate COVID cases (MP), and the healthy donors

a negative impact on $\mathrm{T}$ cell functions, and $\mathrm{T}$ cell proliferation is in particular dependent on arginine supply [28, 29]. Functional assessment of COVID-19 patient $\mathrm{T}$ cells accordingly showed a decreased ability to proliferate in vitro, which predominated for CD8 T cells, although no difference was found between both groups of COVID-19 patients (Fig. 4B). Noteworthy, ability to proliferate in vitro was significantly decreased at admission in ARDS patients for CD4 and CD8 T cells (CD4 T cell: HD vs COVID ARDS D0: 88.12 vs $80.9 ; p=0.01$; CD8 T cell: HD vs COVID ARDS D0: 86.45 vs $77.57 ; p=0.03$ ) meanwhile a significant decrease was observed only for CD8 T cells in patients with moderate pneumonia (CD4 T cell: HD vs COVID MP D0: 88.12 vs $86.68 ; p=0.26$; CD8 T cell: HD vs COVID MP D0: 86.45 vs $70.94 ; p=0.01)$. An increased proportion of apoptotic $\mathrm{T}$ cells among COVID-19 patients compared to healthy donors (HD) was also observed (Fig. 4C). Interestingly, PD-1 and PDL1 exhaustion markers were also more strongly expressed in $\mathrm{T}$ cells and monocytes, respectively, without any relationship with COVID severity (Supplementary Fig. 4). In order to validate the potential role of arginine deprivation in the $\mathrm{T}$ cell proliferation defect, PBMC from ARDS patients were further cultured under L-Arginine supplementation. This supplementation partially but significantly restored the ability for $\mathrm{T}$ cell to proliferate. In contrast, neither the addition of IDO inhibitor nor the addition of anti PD-L1 produced any restoration of T cell proliferation (Fig. 4D).
(HD). Box and whiskers plot features are as follows: central line in the box is the median, bottom line of the box is first quartile (25\%), and top line of box is third quartile ( $75 \%)$. Bottom of whiskers is minimum value; top of whiskers is maximum value. Groups were compared using Kruskal Wallis test with Dunn's multiple comparison test. $* P<0.05$; $* * P<0.01$; $* * * P<0.001 ; * * * * P<0.0001$

\section{Discussion}

Severe forms of COVID-19 with ARDS have been associated with biological and clinical markers of acquired immunosuppression such as opportunistic bacterial infection, virus reactivation, and invasive aspergillosis $[1,11,30]$. This immunosuppression constantly involves lymphopenia and an unbalanced innate vs adaptive immune response, thus delaying pathogen clearance and promoting lung injury. However, the basis for such a dysregulation remain poorly understood [5, 21, 31]. We found that COVID-19 was significantly associated with lymphopenia at admission, mostly affecting CD8 EM cells, and that $\mathrm{T}$ cell defects were correlated with M-MDSC expansion and decreased arginine level.

Reduced T cell number persisted during the first week after admission among ARDS patients, confirming that lymphopenia is a marker of severity in COVID-19 [6, 32]. CD8 T cells are essential for controlling the spread of the virus and for limiting pulmonary damage and mortality, by eliminating infected epithelial cells, thus decreasing virus replication [29, 33]. Autopsies of some patients with COVID-19 pneumonia have evidenced interstitial inflammatory infiltrates dominated by lymphocytes, suggesting that lymphopenia may result from the migration of $\mathrm{T}$ cells to injured tissues [34]. Whereas infected $\mathrm{T}$ cells by SARS-CoV-2 has been 

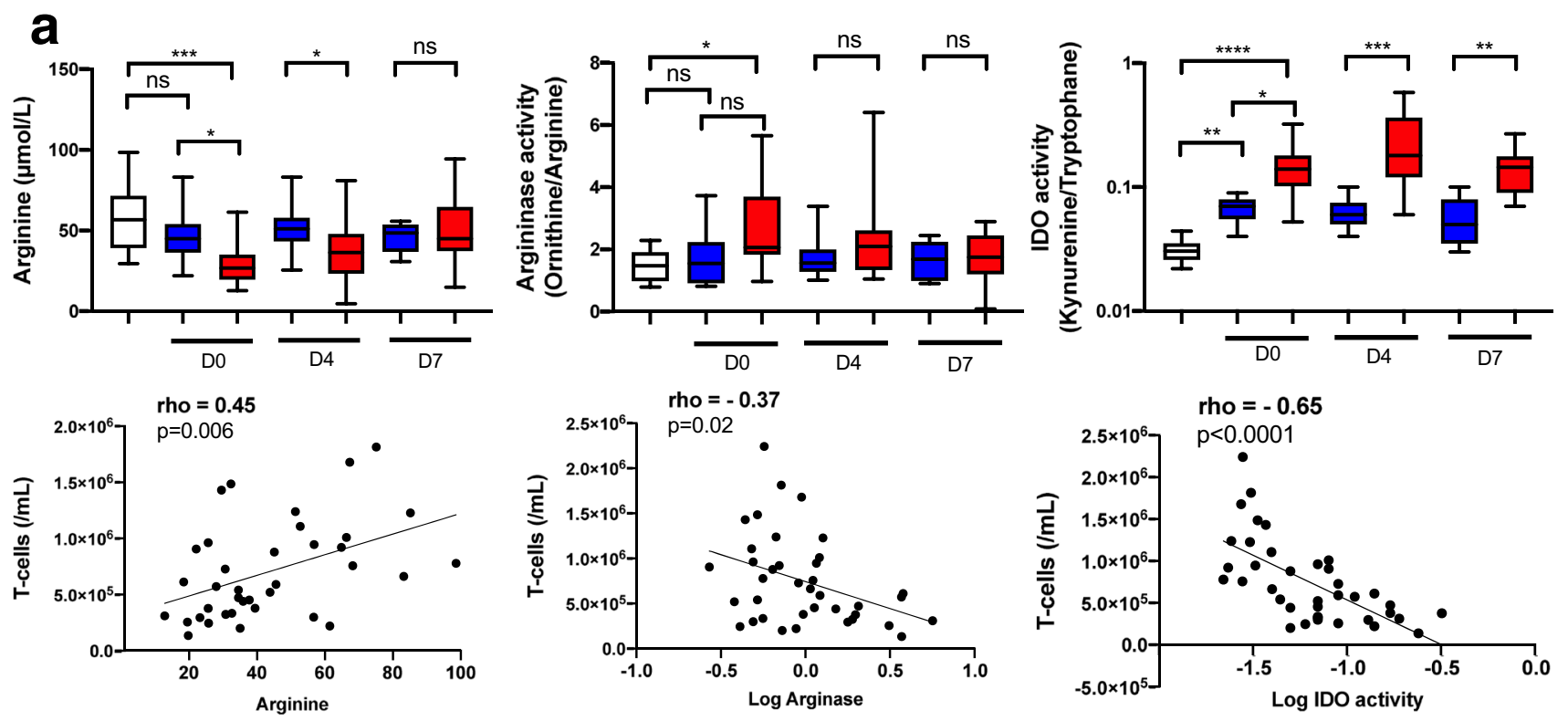

$\square$ HD $\square$ Moderate Pneumonia

ARDS

b

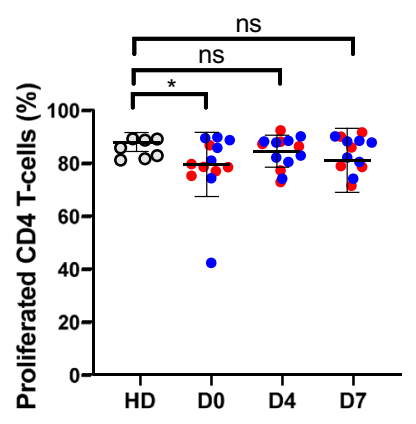

HD

Moderate Pneumonia

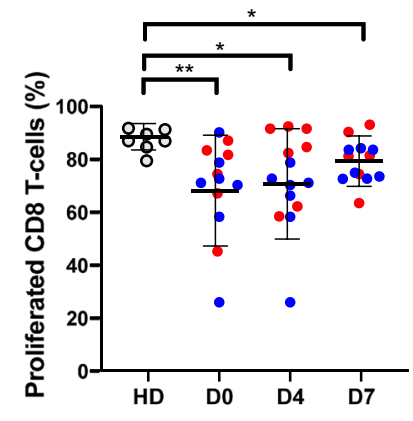

C

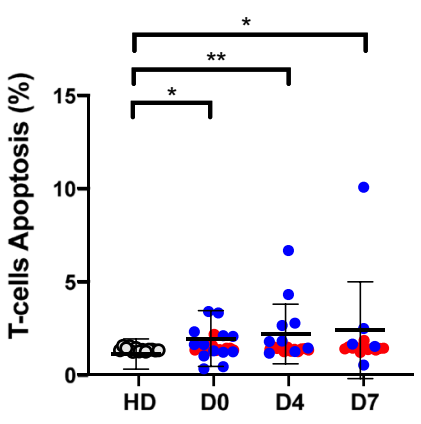

d
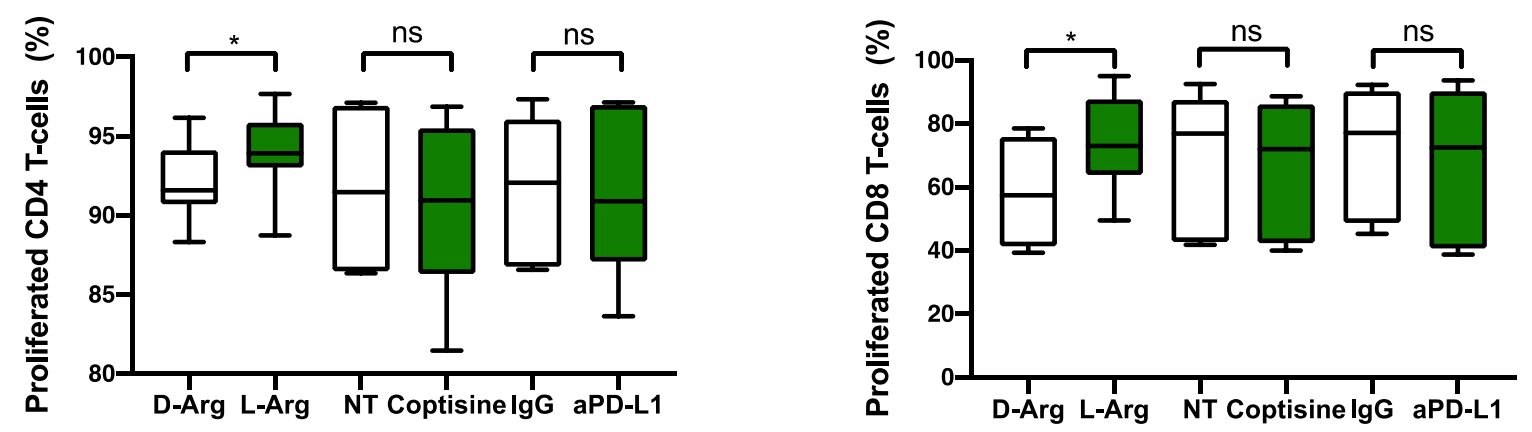

Control

Treatment

documented, it is nevertheless unclear whether a direct cytotoxic effect might contribute to lymphopenia, unlike MERS$\mathrm{CoV}$ infection, in which the virus directly binds its receptor to
T cells $[8,9]$. Our findings suggest that the diminished ability of lymphocytes to proliferate and the enhanced apoptosis are among contributing factors of change in lymphocyte count at 
Fig. 4 SARS-CoV-2 ARDS induces a significant decrease in plasma arginine concentration; its supplementation restores the ability of T-cells to proliferate in-vitro. A Plasma arginine, ornithine, kynurenine, and tryptophan concentrations were measured by liquid chromatography coupled with tandem mass spectrometry among 13 healthy donors (HD), 13 patients hospitalized for SARS-CoV-2 moderate pneumonia (MP), and 13 patients hospitalized for SARS-CoV-2 ARDS (ARDS) $24 \mathrm{~h}$ after their admission (D0), 4 days after (D4), and 7 days after (D7). Arginase activity was calculated using the ornithine/arginine ratio and IDO activity was calculated using the kynurenine/tryptophan ratio. B PBMC obtained at admission (D0), 4 days after admission (D4), and 7 days after (D7) from 14 patients hospitalized for a SARS-CoV-2 infection and from $7 \mathrm{HD}$ were stimulated with anti-CD3/anti-CD28 monoclonal antibodies after CFSE labelling. The proportion of proliferated T-cells were determined by flow cytometry. C Proportion of apoptotic $\mathrm{T}$ cells was determined by flow cytometry using a Caspase-3 staining among 26 patients hospitalized for a SARS-CoV-2 infection at admission (D0), 4 days after (D4), and 7 days after (D7) and from $11 \mathrm{HD}$. Results are expressed by the percentage of Caspase $3^{\text {pos }} \mathrm{T}$ cells. D PBMC obtained from 7 patients hospitalized for a SARS-CoV-2 ARDS were stimulated with anti-CD3/anti-CD28 monoclonal antibodies after CFSE labelling. Culture media were enriched with either L-arginine (L-Arg) or with control D-arginine (D-arg), IDO inhibitor (Coptisine) or vehicle (NT), PD-L1 biding antibody (aPD-L1), or its isotype (IgG). The proportion proliferated $\mathrm{T}$ cells were determined by flow cytometry. Box and whiskers plot features are as follows: central line in the box is the median, bottom line of the box is first quartile (25\%), and top line of box is third quartile (75\%). Bottom of whiskers is minimum value; top of whiskers is maximum value. Groups were compared using Kruskal Wallis test with Dunn's multiple comparison test (A), Mann-Whitney $U$ test $(\mathbf{A}, \mathbf{B}$, and $\mathbf{C})$ or Wilcoxon test (D) as appropriate. Pearson correlation coefficients (rho) and $P$ values are indicated for each correlation (a). $* P<0.05 ; * * P<0.01 ; * * * P<0.001$

admission. The loss of normal $\mathrm{T}$ cell function could be related to the MDSC expansion observed in the most severe forms of COVID-19 with ARDS. Human MDSC belong to two major types: granulocytic (G-MDSC, Lin ${ }^{\text {eg }}$ HLA$\mathrm{DR}^{\text {neg }} \mathrm{CD} 33^{\text {pos }} \mathrm{CD} 11 \mathrm{~b}^{\text {pos }}$ ) and monocytic (M-MDSC, CD14 ${ }^{\text {pos }}$ HLA-DR ${ }^{\text {low }}$ ) [17]. Because monocyte and neutrophil counts were increased among ARDS patients and were associated with a decrease in effector memory $\mathrm{T}$ cells, we wondered whether an accumulation of circulating MDSC was present among COVID-19 patients. The sustained expansion of plasma MDSC in the first week of hospitalization observed among COVID-19 patients was more pronounced in patients with COVID-19-associated ARDS compared to patients with moderate COVID-19 pneumonia. These cells considerably impair the adaptive immune system by suppressing CD4 and CD8 $\mathrm{T}$ cell activation and function, and they have been associated with infection and mortality among septic patients $[18$, 19]. As found in the present study, up to $50 \%$ of COVID-19associated ARDS had a diagnosis of nosocomial infections (ventilator associated pneumonia) during their ICU stay with opportunistic pathogens or viral reactivations. These infections highlight the profound and sustained acquired immunosuppression among COVID-19-associated ARDS patients which has previously been described among patients admitted for septic shock $[12,18]$.

In line with the increase in circulating MDSC, cytokines involved in their recruitment and expansion (IL-6, CCL2, and G-CSF) were found at higher concentrations in the plasma in the most severe forms of COVID-19 [35]. Interestingly, an increase in cytokines involved in the chemo-attraction of activated T cells (CXCL9, CXCL10), myeloid cells (CXCL9, CXCL10, CCL2), and monocyte polarization (CXCL9) was detected among ARDS patients compared to patients with moderate pneumonia, even at ICU admission, suggesting a migration of myeloid and $\mathrm{T}$ cells from peripheral blood to tissue [34]. Additionally, both inflammatory (IL-6) and immunosuppressive (IL-10) cytokines were markedly present among ARDS patients, the latter one being one of the major mechanisms of immune suppression of MDSC [19]. It is worth noting that both subsets of MDSC are released from the bone marrow following various inflammatory or infectious stimuli, such as IL-6 or G-CSF, which were found at higher concentrations in the plasma in the most severe forms of COVID-19 [5, 11, 36]. However, the suppressive activity of MDSC requires not only factors that promote their expansion but also factors that induce MDSC activation such as IL10 [19]. Several studies support the hypothesis that M-MDSC could be involved in viral infection chronicity and also in acute infections decreasing virus clearance and causing lung injury [36]. Our finding highlights the important role of cytokines in COVID-19 and may support cytokine-targeted therapy to correct the profound defect in host immunity [37, 38].

Early M-MDSC expansion in COVID-19-associated ARDS was associated with a decreased number of $T$ cells that could be explained by a decreased ability of lymphocytes to proliferate since $\mathrm{T}$ cell apoptosis was barely enhanced. This decreased ability of lymphocytes to proliferate has already been found among critically ill septic patients and correlated to a decreased availability of arginine [17]. Additionally, IDO activity was increased at admission among COVID-19 patients and associated with disease severity. IDO is an intracellular, non-secreted enzyme, which catabolizes the degradation of tryptophan into kynurenine. Tryptophan catabolites have immunomodulating effects, leading to the inhibition of $\mathrm{T}$ cell activation, $\mathrm{T}$ cell death, and suppression of $\mathrm{T}$ cell effector functions [39]. Besides interferon- $\gamma$, which is the most important inducer of IDO, IL-6 can also stimulate IDO expression by immune cells and MDSC [19, 39]. Although the relationship between COVID-19 and IDO activity has not been studied yet, enhanced IDO activity appears to be associated with lymphopenia as illustrated by the correlation found in our study and should be suspected as a worsening parameter in COVID-19 pneumonia.

Therapeutic interventions principally focus on targeting the virus directly or diminishing the viral load in order to decrease disease severity and ICU admissions [31, 32]. However, 
adjuvant therapeutic interventions are needed to limit the dysfunctional immune response observed among COVID-19 patients and to decrease the late mortality observed in ICU due to persistent pulmonary inflammation and nosocomial infections acquisition [40]. Arginine administration has been found to maintain immune homeostasis, particularly with respect to $\mathrm{T}$ cells and macrophage functions and a reversal of the alteration in $\mathrm{T}$ cell function associated with trauma or surgery has been demonstrated among patients receiving arginine-enriched diet $[41,42]$. Although there are no convincing results in patients with sepsis [43], restoring arginine availability could restore normal $\mathrm{T}$ cell function including their ability to proliferate, as we found in the present study.

Our study has several limitations. Firstly, only a few patients were included and we did not conduct sample size calculation. This methodological issue could weaken our conclusions. Secondly, we did not provide mechanistic information on how arginine could restore $\mathrm{T}$ cell function. Although the immunosuppressive effects of MDSC mediated through arginine depletion and lymphocyte mitochondrial dysfunction have been extensively studied in malignancies [28], mechanistic approaches should be performed in COVID-19 patients to strengthen our conclusions and develop therapeutic trials. Lastly, since T cell subpopulations differ in their proliferation rates and metabolic profile when activated, providing data regarding the capacity of purified COVID-19 $\mathrm{T}$ cell subsets to proliferate would be mandatory to definitively conclude on the qualitative versus quantitative $\mathrm{T}$ cell alterations $[28,44,45]$.

\section{Conclusion}

To summarize, COVID-19 is responsible for MDSC expansion and $\mathrm{T}$ cell dysfunction, mainly CD8 $\mathrm{T}$ cells, through arginine depletion. This dysfunction is sustained in ARDS patients, the most severe form of COVID-19. Restoring arginine availability could be of value to restore lymphocyte function and prevent patients from acquiring nosocomial infections during their ICU stay.

Supplementary Information The online version contains supplementary material available at https://doi.org/10.1007/s10875-020-00920-5.

Author Contributions Conception and design was provided by FR, ML, MG, MR, MC, KT, and JMT. Experiments, data analysis, and interpretation were carried out by FR, ML, MG, KP, AG, MaL, CV, DR, SLG, $\mathrm{JD}, \mathrm{CM}, \mathrm{CB}, \mathrm{MR}, \mathrm{MC}, \mathrm{KT}$, and JMT. Patient inclusions were performed by AM, BP, CC, YLT, MRe, ALB, AB, BCR, TL, and YL. CT scan analyses were performed by MLed. Drafting and revision of the manuscript were carried out by FR, ML, MG, MR, MC, KT, and JMT.

Funding This work was supported by the CFTR $^{2}$ (COVID Fast Track Research Rennes) grant from the University hospital of Rennes, France (FR, ML). MC was supported by a grant from Fondation pour la Recherche Médicale and Agence Nationale de la Recherche (ANR,
Flash CoViD « HARMONICOV ») grant. JMT was supported by a Fondation de l'Avenir grant (Prix des donateurs, Mutualité Fonction Publique).

\section{Compliance with Ethical Standards}

Conflict of Interest The authors declare that they have no competing interests

\section{References}

1. Grasselli G, Zangrillo A, Zanella A, Antonelli M, Cabrini L, Castelli A, et al. Baseline characteristics and outcomes of 1591 patients infected with SARS-CoV-2 admitted to ICUs of the Lombardy region, Italy. JAMA. 2020;323:1574-81.

2. Zhou F, Yu T, Du R, Fan G, Liu Y, Liu Z, et al. Clinical course and risk factors for mortality of adult inpatients with COVID-19 in Wuhan, China: a retrospective cohort study. Lancet. 2020;395: 1054-62.

3. Wu C, Chen X, Cai Y, Xia J, Zhou X, Xu S, et al. Risk factors associated with acute respiratory distress syndrome and death in patients with coronavirus disease 2019 pneumonia in Wuhan, China. JAMA Intern Med. 2020;180:934-43.

4. Du RH, Liang LR, Yang CQ, Wang W, Cao TZ, Li M, et al. Predictors of mortality for patients with COVID-19 pneumonia caused by SARS-CoV-2: a prospective cohort study. Eur Respir J. 2020.

5. Tay MZ, Poh CM, Renia L, MacAry PA, Ng LFP. The trinity of COVID-19: immunity, inflammation and intervention. Nat Rev Immunol. 2020;20:363-74.

6. Chen G, Wu D, Guo W, Cao Y, Huang D, Wang H, et al. Clinical and immunologic features in severe and moderate coronavirus disease 2019. J Clin Invest. 2020;130:2620-9.

7. Qin C, Zhou L, Hu Z, Zhang S, Yang S, Tao Y, et al. Dysregulation of immune response in patients with COVID-19 in Wuhan, China. Clin Infect Dis. 2020.

8. Walls AC, Park YJ, Tortorici MA, Wall A, McGuire AT, Veesler D. Structure, function, and antigenicity of the SARS-CoV-2 spike glycoprotein. Cell. 2020;181:281-92 e286.

9. Fung TS, Liu DX. Human coronavirus: host-pathogen interaction. Annu Rev Microbiol. 2019;73:529-57.

10. Zhao J, Zhao J, Legge K, Perlman S. Age-related increases in PGD(2) expression impair respiratory DC migration, resulting in diminished $\mathrm{T}$ cell responses upon respiratory virus infection in mice. J Clin Invest. 2011;121:4921-30.

11. Ruan Q, Yang K, Wang W, Jiang L, Song J. Clinical predictors of mortality due to COVID-19 based on an analysis of data of 150 patients from Wuhan, China. Intensive Care Med. 2020.

12. Leijte GP, Rimmele T, Kox M, Bruse N, Monard C, Gossez M, et al. Monocytic HLA-DR expression kinetics in septic shock patients with different pathogens, sites of infection and adverse outcomes. Crit Care. 2020;24:110.

13. Gattinoni L, Coppola S, Cressoni M, Busana M, Rossi S, Chiumello D. Covid-19 does not Lead to a "typical" acute respiratory distress syndrome. Am J Respir Crit Care Med. 2020;201: 1299-300.

14. Le Balc'h P, Pinceaux K, Pronier C, Seguin P, Tadie JM, Reizine F. Herpes simplex virus and cytomegalovirus reactivations among severe COVID-19 patients. Crit Care. 2020;24:530.

15. Perez-Padilla R, de la Rosa-Zamboni D, Ponce de Leon S, Hernandez M, Quinones-Falconi F, Bautista E, et al. Pneumonia and respiratory failure from swine-origin influenza A (H1N1) in Mexico. N Engl J Med. 2009;361:680-9. 
16. Gey A, Tadie JM, Caumont-Prim A, Hauw-Berlemont C, Cynober L, Fagon JY, et al. Granulocytic myeloid-derived suppressor cells inversely correlate with plasma arginine and overall survival in critically ill patients. Clin Exp Immunol. 2015;180:280-8.

17. Uhel F, Azzaoui I, Gregoire M, Pangault C, Dulong J, Tadie JM, et al. Early expansion of circulating granulocytic myeloid-derived suppressor cells predicts development of nosocomial infections in patients with sepsis. Am J Respir Crit Care Med. 2017;196:315-27.

18. Venet F, Monneret G. Advances in the understanding and treatment of sepsis-induced immunosuppression. Nat Rev Nephrol. 2018;14: 121-37.

19. Veglia F, Perego M, Gabrilovich D. Myeloid-derived suppressor cells coming of age. Nat Immunol. 2018;19:108-19.

20. Dorhoi A, Glaria E, Garcia-Tellez T, Nieuwenhuizen NE, Zelinskyy G, Favier B, et al. MDSCs in infectious diseases: regulation, roles, and readjustment. Cancer Immunol Immunother. 2019;68:673-85.

21. Bordoni V, Sacchi A, Cimini E, Notari S, Grassi G, Tartaglia E, et al. An inflammatory profile correlates with decreased frequency of cytotoxic cells in COVID-19. Clin Infect Dis. 2020.

22. Agrati C, Sacchi A, Bordoni V, Cimini E, Notari S, Grassi G, et al. Expansion of myeloid-derived suppressor cells in patients with severe coronavirus disease (COVID-19). Cell Death Differ. 2020;27: 3196-207.

23. Force ADT, Ranieri VM, Rubenfeld GD, Thompson BT, Ferguson ND, Caldwell E, et al. Acute respiratory distress syndrome: the Berlin definition. JAMA. 2012;307:2526-33.

24. Blot SI, Taccone FS, Van den Abeele AM, Bulpa P, Meersseman $\mathrm{W}$, Brusselaers $\mathrm{N}$, et al. A clinical algorithm to diagnose invasive pulmonary aspergillosis in critically ill patients. Am J Respir Crit Care Med. 2012;186:56-64

25. Le Gall JR, Lemeshow S, Saulnier F. A new simplified acute physiology score (SAPS II) based on a European/North American multicenter study. JAMA. 1993;270:2957-63.

26. Lambden S, Laterre PF, Levy MM, Francois B. The SOFA scoredevelopment, utility and challenges of accurate assessment in clinical trials. Crit Care. 2019;23:374.

27. Camus C, Bellissant E, Sebille V, Perrotin D, Garo B, Legras A, et al. Prevention of acquired infections in intubated patients with the combination of two decontamination regimens. Crit Care Med. 2005;33:307-14.

28. Geiger R, Rieckmann JC, Wolf T, Basso C, Feng Y, Fuhrer T, et al. L-arginine modulates $\mathrm{T}$ cell metabolism and enhances survival and anti-tumor activity. Cell. 2016;167:829-42 e813.

29. Wherry EJ. T cell exhaustion. Nat Immunol. 2011;12:492-9.

30. van Arkel ALE, Rijpstra TA, Belderbos HNA, van Wijngaarden P, Verweij PE, Bentvelsen RG. COVID-19 associated pulmonary aspergillosis. Am J Respir Crit Care Med. 2020.
31. Li H, Liu L, Zhang D, Xu J, Dai H, Tang N, et al. SARS-CoV-2 and viral sepsis: observations and hypotheses. Lancet. 2020;395:151720.

32. Merad M, Martin JC. Pathological inflammation in patients with COVID-19: a key role for monocytes and macrophages. Nat Rev Immunol. 2020.

33. Jozwik A, Habibi MS, Paras A, Zhu J, Guvenel A, Dhariwal J, et al. RSV-specific airway resident memory CD8+ T cells and differential disease severity after experimental human infection. Nat Commun. 2015;6:10224.

34. Xu Z, Shi L, Wang Y, Zhang J, Huang L, Zhang C, et al. Pathological findings of COVID-19 associated with acute respiratory distress syndrome. Lancet Respir Med. 2020;8:420-2.

35. Gabrilovich DI, Nagaraj S. Myeloid-derived suppressor cells as regulators of the immune system. Nat Rev Immunol. 2009;9:16274.

36. Goh C, Narayanan S, Hahn YS. Myeloid-derived suppressor cells: the dark knight or the joker in viral infections? Immunol Rev. 2013;255:210-21.

37. Remy KE, Mazer M, Striker DA, Ellebedy AH, Walton AH, Unsinger $\mathrm{J}$, et al. Severe immunosuppression and not a cytokine storm characterizes COVID-19 infections. JCI Insight. 2020;5.

38. Luo Y, Mao L, Yuan X, Xue Y, Lin Q, Tang G, et al. Prediction model based on the combination of cytokines and lymphocyte subsets for prognosis of SARS-CoV-2 infection. J Clin Immunol. 2020;40:960-9.

39. Munn DH, Mellor AL. Indoleamine 2,3 dioxygenase and metabolic control of immune responses. Trends Immunol. 2013;34:137-43.

40. Hall MW, Joshi I, Leal L, Ooi EE. Immune modulation in COVID19: strategic considerations for personalized therapeutic intervention. Clin Infect Dis. 2020.

41. Heyland DK, Novak F, Drover JW, Jain M, Su X, Suchner U. Should immunonutrition become routine in critically ill patients? A systematic review of the evidence. JAMA. 2001;286:944-53.

42. Tepaske R, Velthuis H, Oudemans-van Straaten HM, Heisterkamp $\mathrm{SH}$, van Deventer SJ, Ince C, et al. Effect of preoperative oral immune-enhancing nutritional supplement on patients at high risk of infection after cardiac surgery: a randomised placebo-controlled trial. Lancet. 2001;358:696-701.

43. Rice TW. Immunonutrition in critical illness: limited benefit, potential harm. JAMA. 2014:312:490-1.

44. Gattinoni L, Speiser DE, Lichterfeld M, Bonini C. T memory stem cells in health and disease. Nat Med. 2017;23:18-27.

45. Kedia-Mehta N, Finlay DK. Competition for nutrients and its role in controlling immune responses. Nat Commun. 2019;10:2123.

Publisher's Note Springer Nature remains neutral with regard to jurisdictional claims in published maps and institutional affiliations. 